\title{
Correction to: PAIT-Survey Follow-Up: Changes in Albuminuria in Hypertensive Diabetic Patients with Mild-Moderate Chronic Kidney Disease
}

\author{
Francesco Fici ${ }^{1}$ Elif Ari Bakir ${ }^{2}$. Elif Ilkay Yüce ${ }^{3} \cdot$ Serdal Kanuncu $^{4} \cdot$ Wim Makel $^{5} \cdot$ Bahar Arican Tarim $^{6}$. \\ Nicolás Roberto Robles ${ }^{1,7,8}$ (D)
}

Published online: 12 January 2021

(c) Italian Society of Hypertension 2021

\section{Correction to:}

High Blood Press Cardiovasc Prev (2020) 27(1):43-49

https://doi.org/10.1007/s40292-020-00358-1

Table 3 should appear as follows:

$* \mathrm{p}<0.001$ vs baseline, [McNemar test]

$* * \mathrm{p}<0.05$ vs baseline, [McNemar test]

$\$ \mathrm{p}<0.05$ vs other groups, [Chi-square test]

The original article can be found online at https://doi.org/10.1007/ s40292-020-00358-1.

Nicolás Roberto Robles nrrobles@yahoo.es

1 Catedra de Riesgo Cardiovascular, Universidad de Salamanca, Salamanca, Spain

2 Department of Nephrology, Bahcesehir University Hospital, Istanbul, Turkey
Table 3 Changes in prevalence of patients with albuminuria with different treatment strategies

\begin{tabular}{|c|c|c|c|c|}
\hline Treatment & $\begin{array}{l}\text { Amlodi- } \\
\text { pine-vals- } \\
\text { artan }\end{array}$ & $\begin{array}{l}\text { Amlodi- } \\
\text { pine-perin- } \\
\text { dopril }\end{array}$ & $\begin{array}{l}\text { Lercanidi- } \\
\text { pine-enal- } \\
\text { april }\end{array}$ & $\begin{array}{l}\text { Verapamil- } \\
\text { trandolapril }\end{array}$ \\
\hline \multicolumn{5}{|c|}{ Microalbuminuria } \\
\hline Before (n) & 77 & 68 & 92 & 26 \\
\hline After (n) & 65 & 60 & 54 & 21 \\
\hline$\Delta(\mathrm{n})$ & -12 & -8 & -38 & -5 \\
\hline$\%$ & -15.6 & -11.8 & -41.3 & $-19.2 * *$ \\
\hline \multicolumn{5}{|c|}{ Macroalbuminuria } \\
\hline Before & 18 & 10 & 16 & 3 \\
\hline After & 12 & 10 & 12 & 3 \\
\hline$\Delta(\mathrm{n})$ & -6 & 0 & -4 & 0 \\
\hline$\%$ & -27.8 & - & -25.0 & - \\
\hline \multicolumn{5}{|c|}{ Normoalbuminuria } \\
\hline Before & 122 & 75 & 133 & 28 \\
\hline After & 140 & 83 & 175 & 33 \\
\hline$\Delta(\mathrm{n})$ & +18 & +8 & +42 & +5 \\
\hline$\%$ & +14.8 & +10.7 & $+28.6^{\$}$ & $+17.8 * *$ \\
\hline
\end{tabular}

Kelkit State Hospital, Gumushane, Turkey

Family Health Center, Defne-Hatay, Turkey

5 Clinical Research Facilities International B.V, Schaijk, The Netherlands

6 Kartal Research and Education Hospital, Istanbul, Turkey

7 Hospital Universitario de Badajoz, Badajoz, Spain

8 Unidad de Hipertensión Arterial, Hospital Infanta Cristina, Carretera de Portugal s/n, 06080 Badajoz, Spain 